Proc. of the XI Int. Conf. - Ion Implantation and other Applications of Ions and Electrons, Kazimierz Dolny 2016

\title{
Modification of MIS Devices by Irradiation and High-Field Electron Injection Treatments
}

\author{
D.V. Andreev ${ }^{a}$, G.G. Bondarenko ${ }^{b}$, V.V. Andreev ${ }^{a, *}$, V.M. Maslovsky ${ }^{c}$ \\ AND A.A. STOLYAROV ${ }^{a}$ \\ ${ }^{a}$ Bauman Moscow State Technical University, Kaluga Branch, 2, Bazhenov St., Kaluga 248000, Russia \\ ${ }^{b}$ National Research University Higher School of Economics, 20, Myasnitskaya St., Moscow 101000, Russia \\ ${ }^{c}$ Zelenograd Research Institute of Physical Problems, 5, Georgievskiy prosp., Zelenograd, Moscow 103460, Russia \\ Methods to modify gate dielectrics of MIS structures by irradiation treatments and high-field electron injection \\ into dielectric are considered. In addition, distinctive features of these methods used to correct parameters of MIS \\ devices are studied. It was found out that negative charge, accumulating in the thin film of phosphosilicate glass \\ (PSG) of the MIS structure having a two-layer gate dielectric $\mathrm{SiO}_{2}-\mathrm{PSG}$ under the high-field injection or during \\ the irradiation treatment can be used to correct the threshold voltage to improve the charge stability and raise the \\ voltage of breakdown for the MIS devices. It is proved that the density of electron traps rises with the increasing \\ thickness of the PSG film. In this paper a method to modify electrophysical characteristics of MIS structures by \\ the high-field tunnel injection of electrons into the gate dielectric under the mode of controlled current stress is \\ proposed. The method allows to monitor changing of MIS structure parameters directly during the modification \\ process.
}

DOI: $10.12693 /$ APhysPolA.132.245

PACS/topics: 72.20.Ht, 72.20.Jv, 73.20.At, 73.40.Qv, 73.40.Ty, 77.22.Jp, 77.55.-g

\section{Introduction}

Nowadays the vast majority of produced semiconductor devices is manufactured based on the MIS structures. One of the major components of MIS devices are the thin dielectric films. This parameter determines characteristics and reliability of MIS structures. An important problem of modern micro- and nanoelectronics is developing of technologies and methods to correct parameters of MIS structures not only during their production, but also during their operation [1-4]. In order to accomplish this, it is necessary to develop new nanosized dielectric films and structures based on them, whose features allow to modify their charge state in a wide range of values and keep that modified charge state for a long time. Multilayer dielectric films, including the $\mathrm{SiO}_{2}$ film doped with phosphorus, are promising for these purposes $[2,4]$.

In this paper we consider a possibility of modifying the MIS structures, having $\mathrm{SiO}_{2}$ doped with phosphorus by the high-field tunnel injection of electrons into the gate dielectric and using crystal irradiation by an electron beam with different energies.

\section{Experimental samples and method of experiment}

The test MIS capacitors based on the thermal $\mathrm{SiO}_{2}$ film and the MIS capacitors based on the two-layer $\mathrm{SiO}_{2}$-PSG gate dielectric as the experimental samples were used.

*corresponding author; e-mail: vladimir_andreev@bmstu.ru
Both types of the MIS capacitors were manufactured on the $n$-type Si substrate. The film of silicon dioxide with 7-50 $\mathrm{nm}$ thickness was created by thermal oxidation of silicon in oxygen atmosphere at $850-1000^{\circ} \mathrm{C}$. The PSG film of 3-15 nm thickness was formed by diffusion of phosphorus from the gas phase by pyrolysis of $\mathrm{POCl}_{3}-\mathrm{O}_{2}$ mixture at $900^{\circ} \mathrm{C}$. In order to obtain experimental samples of different thickness of PSG film, the time of phosphorus predeposition was varied in the range of $1.5-6 \mathrm{~min}$. Then in order to finish formation of the PSG film, the wafers were annealed for $15 \mathrm{~min}$ at $1000^{\circ} \mathrm{C}$. The polysilicon $\left(\mathrm{Si}^{*}\right)$ films doped with the phosphorus and aluminum films with the area of $10^{-4}-10^{-2} \mathrm{~cm}^{2}$ were used as gates.

In order to make the injection modification of MIS structures, high-field tunnel injection of electrons from the substrate [1-4] was applied in the mode of flowing of constant injection current with the density of $0.1 \mu \mathrm{A} / \mathrm{cm}^{2}$ to $10 \mathrm{~mA} / \mathrm{cm}^{2}$. During the injection, monitoring of voltage dropped on the MIS structure was applied. It allows to acquire information about change of the dielectric charge state directly from modification. In order to determine a value of thermostable part of accumulated negative charge, the MIS structures were annnealed at $150-250{ }^{\circ} \mathrm{C}$ during $5-30 \mathrm{~min}$ after the injection and radiation treatments.

At the modification time, the charge state of MIS structures was monitored using the $C-V$ and of multiplelevel current stress methods [5,6]. During the high-field injection under the constant current stress, an increment of voltage $\Delta V_{I}$ dropped on the MIS structure that characterizes change of sample charge state was observed [4-6].

In order to study influence of the electron irradiation treatments on the characteristics of MIS structures, there 
was used the Zeiss EVO 40 scanning electron microscope and particle accelerator. Using the microscope it was possible to radiate the MIS structures with the electrons having the energies in the range from 15 to $20 \mathrm{keV}$ and the value of current beam of $I=8 \mathrm{nA}$ with the fluence of $5 \times 10^{14} \mathrm{~cm}^{-2}$. Using the particle accelerator there were radiated the MIS structures by the electrons with the energies of $2 \mathrm{MeV}$ and the value of current beam of $I=50 \mu \mathrm{A}$ with the fluence $\Phi$ in the range from $10^{12}$ to $10^{14} \mathrm{~cm}^{-2}$.

\section{Experimental results and discussion}

Figure 1 shows the dependences of increment of the voltage dropped on the MIS structure during injection (1) and irradiation $(2,3)$ modifications on the density of injected charge and electron fluence, respectively. In order to realize the mode of high-field injection of electrons from the silicon substrate, the impulse of constant current with the density of $1 \mu \mathrm{A} / \mathrm{cm}^{2}$ was applied. In Fig. 1 the density of injected electrons is defined as $N=Q_{\text {inj }} / q$, where $Q_{\text {inj }}$ is the charge injected into the dielectric, $q$ is the elementary charge.

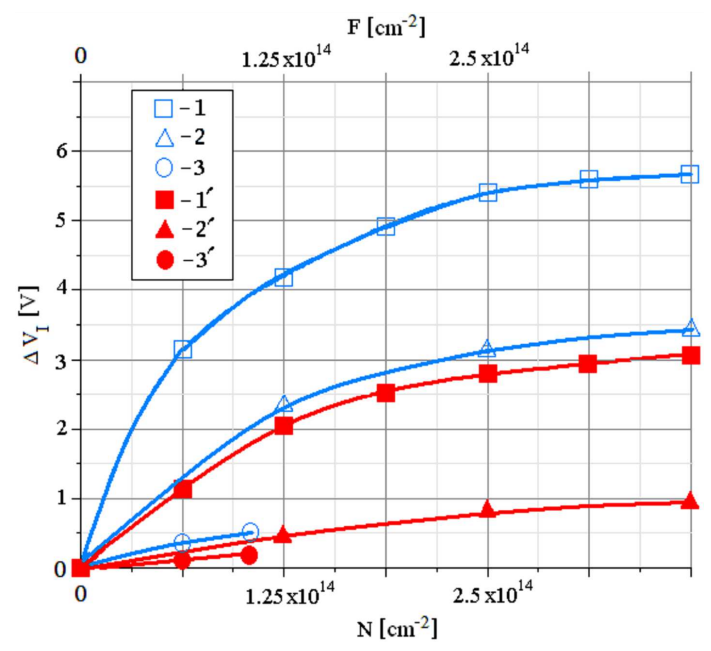

Fig. 1. Dependences of increment of the voltage dropping on the MIS structure at high-field injection of electrons (1) and irradiation by electrons with the energies of $18 \mathrm{keV}(2), 2 \mathrm{MeV}(3)$ as well as after annealing of the treated samples at $200^{\circ} \mathrm{C}\left(1^{\prime}, 2^{\prime}, 3^{\prime}\right)$, on density of the injected electrons $(N)\left(1,1^{\prime}\right)$ and the fluence of electrons $(F)$ during irradiation.

Figure 2 shows the dependences of an increment of the voltage, dropping on the MIS structure, which is stipulated for an integral charge located at the surface states under the injection of electrons (1) and electron irradiation with the energy of $18 \mathrm{keV}(2)$ and $2 \mathrm{MeV}$ (3) on the density of injected electrons $(N)(1)$ and the fluence of electrons $(F)$ when radiating (curves 2,3 ). These dependences were obtained by acquisition of $C-V$ data provided by interruption of the irradiation process.

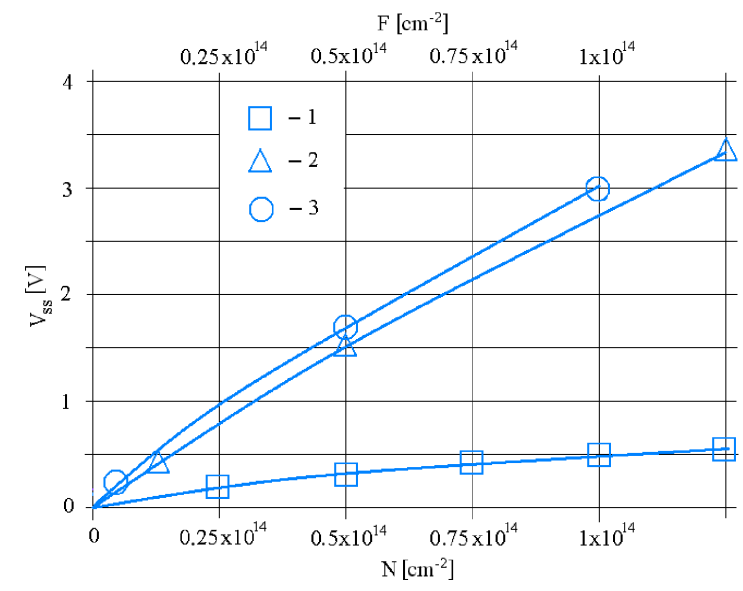

Fig. 2. Dependences of increment of the voltage dropping on the MIS structure, which is conditioned on the integral charge located at the surface states, when the high-field injection of electrons (1) or electron irradiation with the energies of $18 \mathrm{keV}(2)$ and $2 \mathrm{MeV}$ (3), on density of the injected electrons $(N)(1)$ and electron the fluence $(F)$ (curves 2,3).

The curves $1^{\prime}, 2^{\prime}$, and $3^{\prime}$ in Fig. 1 characterize the thermostable component of the negative charge. In order to obtain this component, after the injection $\left(1^{\prime}\right)$ and radiation treatments $\left(2^{\prime}, 3^{\prime}\right)$ the MIS structures were annealed for $20 \mathrm{~min}$ at $200^{\circ} \mathrm{C}$. It follows from Fig. 1 that the thermostable component of negative charge during the injection modification (curve $1^{\prime}$ ), is higher than the similar charge, which taking place when the electron irradiation (curves $2^{\prime}, 3^{\prime}$ ). Therefore, the injection modification is more effective and under some conditions it leads to less degradation of MIS structures [7]. However, the electron irradiation allows to perform group treatment of the crystals located at a semiconductor wafer. This feature significantly enhance treatment productivity. Figure 1 demonstrates that for the irradiation modification of MIS structures the more effective way is to use the irradiation by low-energy electrons (Fig. 1, curves 2 and $2^{\prime}$ ) at which the PSG film accumulates higher density of the negative charge with respect to the irradiation by high-energy electrons.

Figure 1 shows that during the electron irradiation, a lower density of negative charge (curves 2, 3, Fig. 1) accumulates in the gate dielectric $\mathrm{SiO}_{2}$-PSG with respect to the case of the high-field injection (curve 1, Fig. 1). Besides, during the irradiation by high-energy electrons (curve 3, Fig. 1), a significantly lower value of the negative charge accumulates in comparison with the case of the low-energy electrons. Apparently, during the electron irradiation, ionization processes taking place in the gate dielectric, have great influence on change of the charge state of MIS structure. Because of the radiation ionization, a considerable number of the electron-hole pairs take place in the dielectric film. However, during the high-field injection, the unipolar transport of electrons 
occurs mostly. Electrons with the energy of $2 \mathrm{MeV}$ exert a substantial effect onto degradation of charge state of MIS structures, probably, due to the presence of the ionization processes in the silicon substrate and occurrence of radiation defects in the MIS structure.

When increasing thickness of the PSG layer, the range of possible modifications of the threshold voltage of MIS based transistors, which are with $\mathrm{SiO}_{2}-\mathrm{PSG}$ gate dielectric becomes wider in both the high-field injection of electrons from the silicon substrate and the electron irradiation. However, in order to provide acceptable values of density of surface states, the amount of the injected charge should not exceed $0.3 \mathrm{mC} / \mathrm{cm}^{-2}$ during correction of the threshold voltage. At the same time, the fluence of electrons during electron irradiation should be less than $10^{13} \mathrm{~cm}^{-2}$. It is also important to note that when increasing the electron beam energy, the maximal possible value of fluence becomes lower. It is advisable to limit a range of the current stress with the values of $10^{-7} \div 10^{-5} \mathrm{~A} / \mathrm{cm}^{2}$ during modification of the charge state of MIS structure. Decrease of current stress amplitude is followed by difficulties of technical realization and is not expedient because of significant increase of injection time of required charge amount. Rising the injected charge density leads to significant growth of sample breakdown possibility and also to augmentation of surface states density at the $\mathrm{Si}-$ $\mathrm{SiO}_{2}$ interface [4] during the electron injection from the Si substrate.

It was established that the range of possible changes $\Delta V_{I}$, in the case of the same value of the injected charge and electron fluence, is wider when rising the thickness of PSG film. It relates both a shift of centroid of the accumulated negative charge in direction to the $\mathrm{Si}-\mathrm{SiO}_{2}$ interface when rising the thickness of PSG film and increasing density of the accumulated negative charge. Hightemperature annealing for $20 \mathrm{~min}$ at $200^{\circ} \mathrm{C}$ leads to draining 40-50\% of negative charge which was accumulated at the time of the high-field injection of electrons or the electron irradiation. Growth of annealing time hardly has any influence on the remaining thermostable component of negative charge. Increase of the PSG film thickness is not acceptable with respect to the thin gate dielectrics since it can result in throughout doping of the $\mathrm{SiO}_{2}$ film by phosphorus, and, consequently, in deterioration of charge stability of the gate dielectric and the semiconductor/dielectric interface. Augmentation of phosphorus concentration inside the PSG film by more than $1.5 \%$ can bring to occurrence of effects such as polarization and reduction of charge stability of gate dielectric $[5,8-$ 10]. Therefore, it is preferable to increase a diapason of correction of electrophysical parameters of MIS devices by changing a ratio between the thicknesses of $\mathrm{SiO}_{2}$ and PSG films and by shifting the centroid of negative charge closer to the $\mathrm{Si}-\mathrm{SiO}_{2}$ interface. Simultaneously, the concentration of phosphorus in PSG film should not exceed $1.5 \%[10]$.

Accumulation of required density of the thermostable component of negative charge inside the gate dielectric

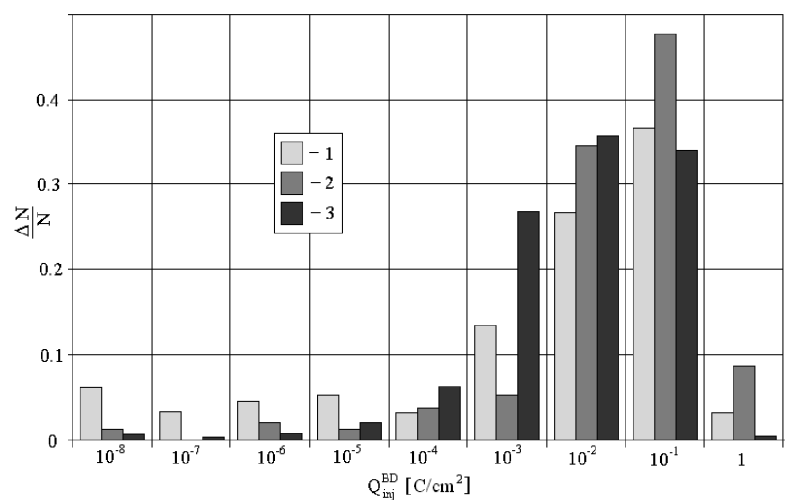

Fig. 3. Histograms of distribution of MIS structures by charge, injected into the dielectric until its breakdown, for the samples with the $\mathrm{SiO}_{2}$ gate dielectric (1) and those with the $\mathrm{SiO}_{2}$-PSG two-layer gate dielectric $(2,3)$ when PSG is of different thickness: $2-9 \mathrm{~nm}, 3-$ $22 \mathrm{~nm}$.

volume allows to correct the threshold voltage of MIS transistor, enhance the breakdown voltage of the gate dielectric and lower a possibility of its breakdown by healing defects ("weak spots") accumulating negative charge in them $[8,10]$. Figure 3 shows the histograms of MIS structures distribution by charge injected into the dielectric until breakdown when the current stress $[1,5]$ for the samples with the $\mathrm{SiO}_{2}$ gate dielectric (1) and those with the $\mathrm{SiO}_{2}-\mathrm{PSG}$ gate dielectric $(2,3)$. Figure 3 shows that the usage of the $\mathrm{SiO}_{2}-\mathrm{PSG}$ two-layer gate dielectric allows to rise the average amount of charge injected into the dielectric until breakdown and to diminish a number of defective structures with low charge-to-breakdown. This effect can be explained by healing of the "weak spots" in the gate dielectric due to accumulation of negative charge inside them. In consequence, negative charge accumulation, local injection currents, flowing at spots of defects and leading to the dielectric breakdown, decrease. A result of injected electron trapping is an augmentation of the potential barrier which prevents from currents localization in the regions of extensive defects at the interface of dielectric with the substrate.

\section{Conclusions}

Negative charge, accumulating in the PSG film in the structures with the $\mathrm{SiO}_{2}-\mathrm{PSG}$ two-layer gate dielectric during both high-field tunnel injection of electron and electron irradiation can be used for the MIS devices modification. The usage of high-field injection of electrons to modify the charge state of MIS structures is more preferable than that of the electron irradiation because during the high-field injection, the individual modification of characteristics of each device is possible. In addition, an advantage of the high-field injection usage is that during certain modes of injection it is feasible to lower the attendant degradation processes. Application of the $\mathrm{SiO}_{2}-\mathrm{PSG}$ two-layer gate dielectric, of the phosphorus 
concentration in the PSG film in the range of $0.4-0.9 \%$, allows to increase the average amount of charge injected into the dielectric until its breakdown and reduces quantity of defective structures with a low value of charge-tobreakdown. This effect can be explained by healing of "weak spots", located in the gate dielectric, due to accumulation of negative charge in the gate dielectric. The accumulation of negative charge results in rising the potential barrier and lowering the local injection currents.

\section{Acknowledgments}

This study was supported by the Ministry of Education and Science of the Russian Federation, state task of the Moscow State Technical University, and the Kaluga Region Administration (grant No. 16-42-400791). Support from the Basic Research Program of the National Research University Higher School of Economics is gratefully acknowledged.

\section{References}

[1] D.V. Andreev, G.G. Bondarenko, V.V. Andreev, A.A. Stolyarov, IOP Conf. Series Mater. Sci. Eng. 110, 012041 (2016).
[2] V.V. Andreev, G.G. Bondarenko, V.M. Maslovsky, A.A. Stolyarov, D.V. Andreev, Phys. Status Solidi C 12, 126 (2015).

[3] S. Lombardo, J.H. Stathis, P. Linder, K.L. Pey, F. Palumbo, C.H. Tung, J. Appl. Phys. 98, 121301 (2005).

[4] V.V. Andreev, G.G. Bondarenko, V.M. Maslovsky, A.A. Stolyarov, Acta Phys. Pol. A 125, 1371 (2014).

[5] V.V. Andreev, G.G. Bondarenko, V.M. Maslovsky, A.A. Stolyarov, IOP Conf. Series Mater. Sci. Eng. 41, 012017 (2012).

[6] V.V. Andreev, G.G. Bondarenko, V.M. Maslovsky, A.A. Stolyarov, D.V. Andreev, Phys. Status Solidi C 12, 299 (2015).

[7] D.V. Andreev, G.G. Bondarenko, A.A. Stolyarov, J. Surf. Investig. X-Ray Synchr. Neutron Techn. 10, 450 (2016).

[8] United States Military Standard MIL-STD883H. Method 1019.8.

[9] G.M. Voronkova, V.D. Popov, G.A. Protopopov, Semiconductors 41, 958 (2007).

[10] V.V. Andreev, G.G. Bondarenko, V.M. Maslovsky, A.A. Stolyarov, Acta Phys. Pol. A 128, 887 (2015). 